\title{
High-energy nonthermal radiation from putative Geminga-like gamma-ray pulsars (Research Note)
}

\author{
Z. Jin ${ }^{1,2}$, Z. J. Jiang ${ }^{1}$, and L. Zhang ${ }^{1}$ \\ ${ }^{1}$ Department of Physics, Yunnan University, Kunming, 650091 Yunnan, PR China \\ e-mail: zjjiang@ynu.edu.cn \\ 2 Department of Physics, Yunnan Normal University, Kunming, 650092 Yunnan, PR China
}

Received 6 September 2007 / Accepted 18 January 2008

ABSTRACT

\begin{abstract}
Aims. The gamma-ray spectra of some unidentified Galactic sources show that the corresponding counterparts are likely gamma-ray pulsars. We investigate the possibility of some Geminga-like gamma-ray pulsars as the counterparts of unidentified sources. Methods. There are five Geminga-like gamma-ray pulsar candidates associated with 3EG J0010+7309, 3EG J1835+5918, 3EG J2020+4017, 3EG J0616-3310, and 3EG J1249-8330. Combining the observed nonthermal X-ray emission detected by Chandra or XMM-Newton and the gamma-ray emission by EGRET, we study the high-energy radiation from these putative pulsars based on the revised vacuum outer gap model.

Results. By comparing the estimated spectra with the observations, we estimated the spin parameters and the inclination angles for these five putative pulsars. The observed spectra of the unidentified gamma-ray sources can be explained by this outer gap model.
\end{abstract}

Key words. pulsars: general - gamma rays: theory - radiation mechanisms: non-thermal

\section{Introduction}

With about 170 unidentified gamma-ray objects in the third EGRET catalogue (Hartman et al. 1999), the nature of the unidentified gamma-ray sources are still open questions in current astrophysics. The following facts have an effect on the current beliefs that most of the unidentified Galactic sources whose gamma-ray spectra resemble those of gamma-ray pulsars are probably radio-loud or radio-quiet gamma-ray pulsars (like Geminga). The facts are: (i) pulsars are the only identified Galactic gamma-ray objects with energy higher than $100 \mathrm{MeV}$; (ii) some young pulsars have been discovered to be spatially associated with unidentified sources (Kramer et al. 2003); and (iii) some unidentified gamma-ray sources have been found to be associated with pulsar wind nebulae (PWNe) or supernova remnants (SNRs). The steady gamma-ray spectra of these sources can be described by a hard power law that breaks at several $\mathrm{GeV}$, similar to the gamma-ray pulsars except the extended emission from SNRs (Thompson et al. 1999).

There are five promising candidates for Geminga-like gamma-ray pulsars that may be the counterparts of 3EG J0010+7309 (Brazier et al. 1998; Halpern et al. 2004), 3EG J1835+5918 (Mirabal \& Halpern 2001; Reimer et al. 2001; Halpern et al. 2002), 3EG J2020+4017 (Brazier et al. 1996), 3EG J0616-3310 and 3EG J1249-8330 (La Palombara et al. 2006). The multiwavelength approach has been applied to searching for the counterparts whose radio signals had not been detected (e.g., La Palombara et al. 2006), and all of the candidates are characterized by extremely high values of the X-ray-tooptical flux ratio $f_{\mathrm{X}} / f_{V}$, similar to observed gamma-ray pulsars.

There are mainly two types of models for describing the high-energy gamma-ray emission from pulsars: one polar cap model (e.g., Daugherty \& Harding 1996; Zhang \& Harding 2000; Muslimov \& Harding 2004), the other outer gap model (e.g., Cheng et al. 1986a, b, hereafter CHR86a, CHR86b; Zhang \& Cheng 1997, hereafter ZC97; Hirotani \& Shibata 2001; Zhang et al. 2004, hereafter Zh04; Hirotani 2007). In the outer gap models, two versions have been proposed: vacuum outer gaps (CHR86a; CHR86b; ZC97) and non-vacuum outer gaps (e.g., Hirotani \& Shibata 2001; Hirotani 2007). Although phaseaveraged properties of high-energy emission from pulsars can be explained well in the framework of these two versions of the outer gap models, it has been shown that the phase-resolved properties can be accounted for in the vacuum outer gap model (Cheng et al. 2000; Takata \& Chang 2007; Tang et al. 2008). In this paper, we will use the vacuum outer gap model to explain high-energy nonthermal radiation from putative Gemingalike gamma-ray pulsars. In such a model, more Geminga-like gamma-ray pulsars are predicted because the different directions of the radio and gamma-ray beams. Based on the outer gap model of ZC97, Zhang \& Cheng (1998) studied high-energy gamma-ray radiation from the radio pulsars associated with some unidentified EGRET gamma-ray sources.

Here we focus on some putative Geminga-like gamma-ray pulsars using the new version of the outer gap model (Zh04). For these sources, we take both the observed X-ray and gamma-ray spectra into account and try to find reasonable spin parameters to reproduce the observed spectra. We have organized our paper as follows. In Sect. 2, we briefly review the outer gap model for phase-averaged X-ray and gamma-ray emission from rotationpowered pulsars. In Sect. 3, we apply the outer gap model to explain the observed X-ray and gamma-ray emission from some putative pulsars, and a brief discussion and conclusion is presented in Sect. 4. 


\section{X-ray and gamma-ray emission in the outer gap model}

In the vacuum outer gap model (ZC97; Zh04), about half of the primary $\mathrm{e}^{ \pm}$in the outer gap will flow back toward the star and lose most of the energy via curvature radiation. The surviving energy of these particles will convert into soft X-ray photons and collide with the high-energy curvature photons in the outer gap to produce enough $\mathrm{e}^{ \pm}$to sustain the existence of outer gaps. According to the pair production condition, we can estimate the fractional size $(f)$ of the outer gap. Generally, the outer gap starts at inner boundary $r_{\text {in }}$, where the last open field lines intersect with the null charge surface (where $\boldsymbol{\Omega} \cdot \boldsymbol{B}=0$ ). In the revised outer gap model (Zh04), the fractional size $f$ is the function of period $P$, magnetic field $B$, inclination angle $\alpha$, and distance $r$ to the pulsar. This can be written as $f(r, P, B)=\eta(\alpha, r, P, B) f_{0}(P, B)$ (see Eq. (39) of Zh04), where $f_{0}(P, B)=5.5 P^{26 / 21} B_{12}^{-4 / 7}$ is the fractional size of the outer gap by ignoring the effect of the inclination angle (ZC97), and where $\eta(\alpha, r P, B)$ contains the effect of inclination angle, which can be estimated by using Eq. (37) of $\mathrm{Zh04}$

The pulsed gamma-ray emission comes from the primary $\mathrm{e}^{ \pm}$pairs accelerated in the outer gap. These high-energy particles move along the curved magnetic field lines and lose energy via the synchro-curvature process. The differential flux at the Earth is given by Eqs. (57) and (58) of ZC97, so we use these equations to estimate the gamma-ray spectra of the putative gammaray pulsars at the average distance $\langle r\rangle$, which can be obtained by Eq. (38) of Zh04. There are still two parameters, where the solid angle of gamma-ray beam $\Delta \Omega$ and the maximum value of curvature radius in units of light cylinder radius $x_{\max }$ need to be determined, which can be estimated by comparing the estimated spectrum with the observed spectrum. Here we adopt the twodimensional vacuum solution and assume a uniform structure in the toroidal direction.

Cheng \& Zhang (1999) studied the multicomponent X-ray emission from rotation-powered pulsars based on the outer gap model of ZC97. In this model, the back-flowing particles lose their energy via curvature radiation, and these high-energy photons move toward the neutron star surface and produce $\mathrm{e}^{ \pm}$pairs in the strong magnetic field. The secondary $\mathrm{e}^{ \pm}$pairs lose energy via synchrotron radiation, which will further converted into the next generation of $\mathrm{e}^{ \pm}$pairs in a stronger magnetic field when they come closer to neutron star, and then a cascade develops. This process causes the nonthermal pulsed X-ray emission. Based on the revised model ( $\mathrm{Zh04}$ ), we took the effects of inclination angle and magnetic field geometry into account and upgraded the model about the nonthermal pulsed X-ray emission from the rotation-powered pulsars (Zhang \& Jiang 2005, 2006). The nonthermal X-ray flux at the Earth can be written as

$$
F_{\mathrm{X}}\left(E_{\mathrm{X}}\right)=\frac{1}{4 \pi d^{2}} L_{\mathrm{X}}^{n}\left(E_{\mathrm{X}}\right) \exp \left(-\sigma\left(E_{\mathrm{X}}\right) N_{\mathrm{H}}\right)
$$

where $d$ is the distance to the pulsar, $\sigma\left(E_{\mathrm{X}}\right)$ the photoelectric cross section of the interstellar medium (Morrison \& McCammon 1983), and $N_{\mathrm{H}}$ the interstellar column density. The non-thermal X-ray luminosity $L_{\mathrm{X}}^{n}$ can be estimated by Eq. (24) of Zhang \& Jiang (2006). Here we use this equation to estimate non-thermal X-ray emission from the putative pulsars.

\section{High-energy radiation from radio-quiet gamma-ray pulsar candidates}

\section{1. $R X J 0007.0+7302$ associated with $3 E G$ J0010+7309}

The unidentified gamma-ray source 3EG J0010+7309 is positionally consistent with the SNR CTA 1 (G119.5+10.2). The steady and hard gamma-ray spectrum of 3EG J0010+7309 suggests that its counterpart is a likely gamma-ray pulsar (Lamb \& Macomb 1997; Brazier et al. 1998). The compact object RX J0007.0+7303, detected by ROSAT at the center of CTA 1 , may be the pulsar that powers the surrounding nebula (Slane et al. 1997). The observations by XMM-Newton (Slane et al. 2004) and Chandra (Halpern et al. 2004) show that RX J0007.0+7303 has the typical properties of young neutron stars whose X-ray spectra can be explained by a two-component (power-law plus blackbody) model. It does not detect any signals of radio or optical emission. Although the X-ray observations support the idea that RX J0007.0+7303 is a young pulsar, it still cannot find any pulsed signals. This pulsar is a likely Geminga-like pulsar. Here we combine the observed X-ray and gamma-ray spectrum to infer the spin parameters of this putative pulsar in the outer gap model (Zh04). The observed X-ray spectrum by Chandra is taken from Halpern et al. (2004), the X-ray spectral index $\Gamma_{\mathrm{X}}=1.6 \pm 0.6$, and the distance is estimated as $d=1.4 \mathrm{kpc}$ (Pineault et al. 1993). The gamma-ray data from 3EG J0010+7309 is taken from Brazier et al. (1998).

In the revised outer gap model (Zh04; Zhang \& Jiang 2006), the X-ray spectrum of a pulsar observed on the Earth can be estimated using Eq. (1). There are three parameters that should be estimated: inclination angle $(\alpha)$, period $(P)$, and magnetic field $(B)$. The observations by XMM-Newton and Chandra show that the expected pulsar is probably a Vela-like pulsar (Halpern et al. 2004). The derived inclination angle from the X-ray observation of the Vela pulsar lies between $\alpha \sim 20^{\circ}-\sim 30^{\circ}$ (Zhang \& Jiang 2006), so we first select $\alpha \sim 25^{\circ}$ to estimate the flux. The $P$ and $B$ relate to the estimated spin-down luminosity, which is roughly estimated as $\dot{E} \gtrsim 6 \times 10^{36} \mathrm{erg} \mathrm{s}^{-1}$ (Halpern et al. 2004). These results give us information needed to find the best fitted parameters. We take the Sedov age of CTA $1, \tau_{\mathrm{S}} \simeq 1.3 \times 10^{4} \mathrm{yr}$ (Slane et al. 2004) as the characteristic age $\left(\tau_{\mathrm{c}}=P / 2 \dot{P}\right.$, where $\dot{P}$ is the period derivative) of the putative pulsar, so when we give a specific $P, B$ can be roughly estimated by relation $B \propto(P \dot{P})^{1 / 2}$. Comparing the calculated spectrum with the observed spectrum, we find that the most likely values are $\alpha=24^{\circ}, P=0.19 \mathrm{~s}$, and $B_{12}=6.8 \mathrm{G}$, where $B_{12}=B / 10^{12} \mathrm{G}$. The comparison of the estimated and observed X-ray spectra is shown in Fig. 1a. Using the above parameters, we estimate the fractional size $f$ at the mean distance $\langle r\rangle$ (Zh04), and then estimate the gamma-ray spectrum at $\langle r\rangle$ by using Eqs. (57) and (58) of ZC97. The solid angle of the gamma-ray emission is assumed as $\Delta \Omega=2.5 \mathrm{sr}$, and the parameter $x_{\max }=2.0$, We can see that the modeled spectrum explains the observation well. We also present the estimated spectra when $P=0.16 \mathrm{~s}$ and $B_{12}=7.8$ (dashed line in Fig. 1a) and $P=0.22 \mathrm{~s}$ and $B_{12}=5.7$ (dotted line in Fig. 1a).

\section{2. $R X J 1836.2+5925$ associated with $3 E G \mathrm{~J} 1835+5918$}

3EG J1835+5918 is the brightest unidentified gamma-ray source detected by EGRET at high Galactic latitude (Hartman et al. 1999). The spectra of 3EG J1835+5918 is characterized by a hard power law with spectral index $-1.7 \pm 0.06$ in the $70 \mathrm{MeV}$ to $4 \mathrm{GeV}$ range and a spectral break or turnover above $4 \mathrm{GeV}$ (Reimer et al. 2001), and its flux does not exhibit 


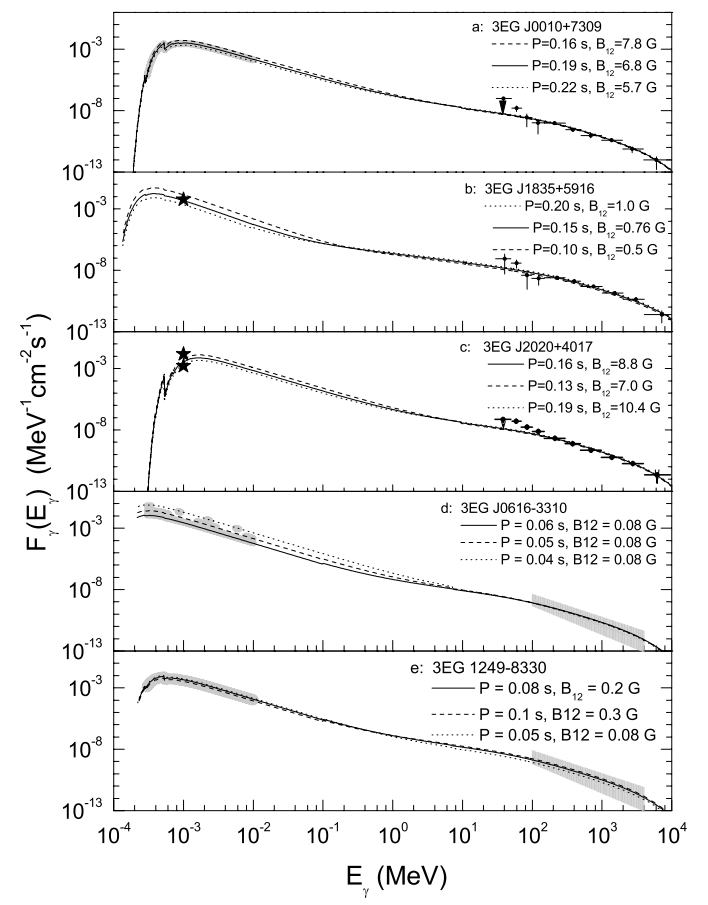

Fig. 1. The non-thermal high-energy emission from five putative Geminga-like gamma-ray pulsars (see text for details).

variability during the EGRET emission. Both of them are similar to those of known gamma-ray pulsars like Vela or Geminga, so the counterpart of 3EG J1835+5918 is likely to be a gammaray pulsar (Reimer et al. 2001). The multifrequency searches find that RX J1836.2+5925 is the likely counterpart of this gamma-ray source, which is most likely a more distant or older Geminga-like gamma-ray pulsar (Mirabal et al. 2000; Mirabal \& Halpern 2001; Reimer et al. 2001; Halpern et al. 2002, 2007).

If RX J1836.2+5925 is born on the Galactic plane, the proper motion shows that the age of RX J1836.2+5925 is at least $\tau_{\mathrm{c}}=6.4 \times 10^{5} \mathrm{yr}$ (Halpern et al. 2007), which can place constraints when we estimate the reasonable spin parameters. The distance to RX J1836.2+5925 is bounded from $250 \mathrm{pc}$ to $800 \mathrm{pc}$, so here we take $d=800 \mathrm{pc}$. The spectral index of the power-law component of the Chandra X-ray spectra can be estimated as $1.6 \leq \Gamma_{\mathrm{X}} \leq 2.8$, and the flux in the $0.2-2 \mathrm{keV}$ is (1.9-2.6) $\times 10^{-14} \mathrm{erg} \mathrm{cm}^{-2} \mathrm{~s}^{-1}$ (Halpern et al. 2002). Based on the above results, we estimate the flux at $1 \mathrm{keV}$ as $\sim 6 \times$ $10^{-6} \mathrm{~cm}^{-2} \mathrm{~s}^{-1} \mathrm{keV}^{-1}$ (star in Fig. 1b), and the column density is estimated as $N_{\mathrm{H}}=4.64 \times 10^{20} \mathrm{~cm}^{-2}$ and $\Gamma_{\mathrm{X}} \sim 2.0$ (Halpern et al. 2002). This estimated $X$-ray flux can give a rough constraint when fitting the observed spectra. In Fig. 1b, we give the estimated nonthermal high-energy spectra by selecting different spin parameters. The inclination angle and gamma-ray beaming solid angle are estimated as $\alpha=30^{\circ}$ and $\Delta \Omega=1 \mathrm{sr}$, respectively. From Fig. 1b, we can see that the estimated spectra from a pulsar with $P=0.15 \mathrm{~s}$ and $B_{12}=0.76 \mathrm{G}$ are consistent with the observed. However, because there are not enough photons to give a precise description of the X-ray spectra, we cannot exclude other parameters, such as $P=0.1 \mathrm{~s}$ and $B_{12}=0.5 \mathrm{G}$, or $P=0.2 \mathrm{~s}$ and $B_{12}=1.0 \mathrm{G}$.

\section{3. $R X$ J2020.2+4026 associated with 3EG J2020+4017}

3EG J2020+4017 is a bright unidentified gamma-ray source located at the center of $\gamma$-Cygni supernova remnant
Table 1. The parameters of the putative pulsars associated with unidentified sources.

\begin{tabular}{llllllll}
\hline \hline 3EG & $\begin{array}{l}P \\
(\mathrm{~s})\end{array}$ & $\begin{array}{l}B_{12} \\
(\mathrm{G})\end{array}$ & $\begin{array}{l}\alpha \\
\left({ }^{\circ}\right)\end{array}$ & $\begin{array}{l}\tau_{\mathrm{c}} \\
\left(10^{5} \mathrm{yr}\right)\end{array}$ & $\begin{array}{l}d \\
(\mathrm{kpc})\end{array}$ & $\begin{array}{l}\Delta \Omega \\
(\mathrm{sr})\end{array}$ & $x_{\max }$ \\
\hline $\mathrm{J} 0010+7309$ & 0.19 & 6.8 & 24 & 0.13 & 1.4 & 2.5 & 2.0 \\
$\mathrm{~J} 1835+5918$ & 0.51 & 0.15 & 30 & 6.4 & 0.8 & 1.0 & 2.0 \\
$\mathrm{~J} 2020+4017$ & 0.16 & 8.8 & 25 & 5.4 & 1.6 & 0.9 & 2.0 \\
$\mathrm{~J} 0616-3310$ & 0.06 & 0.08 & 25 & 90 & 0.4 & 3.0 & 2.5 \\
& 0.05 & 0.08 & 25 & 93 & 0.4 & 3.0 & 2.5 \\
& 0.04 & 0.08 & 25 & 41 & 0.4 & 3.0 & 2.5 \\
$\mathrm{~J} 1249-8330$ & 0.08 & 0.2 & 25 & 25 & 0.4 & 3.0 & 2.5 \\
\hline
\end{tabular}

(SNR G78.2+21) (Hartman et al. 1999). The EGRET flux of 3EG J2020+4017 is steady, and the spectrum can be described by a hard power law with spectral index $\sim-1.9$ at the range of $100 \mathrm{MeV}$ to $2 \mathrm{GeV}$ and a spectral break at about $4 \mathrm{GeV}$ (Merck et al. 1996; Brazier et al. 1996), the counterpart of this source is likely a gamma-ray pulsar. However, it is impossible to find a single $\mathrm{X}$-ray point source as a potential neutron star for producing the gamma-ray radiation (Becker et al. 2004; Weisskopf et al. 2006). Weisskopf et al. (2006), when continuing their search (Becker et al. 2004) for X-ray counterparts to 3EG J2020+4017 using Chandra, find 10 sources as the potential counterpart. For these 10 optically unidentified $\mathrm{X}$-ray sources, the maximum and minimum values of the fluxes at $1 \mathrm{keV}$ are $14.6 \pm 2.2 \times 10^{-6} \mathrm{~cm}^{-2} \mathrm{~s}^{-1} \mathrm{keV}^{-1}$ and $1.6 \pm 0.7 \times 10^{-6} \mathrm{~cm}^{-2} \mathrm{~s}^{-1} \mathrm{keV}^{-1}$ (stars in Fig. 1c), corresponding to S25 and S19, respectively (Weisskopf et al. 2006). If one of them is the putative pulsar that corresponds to 3EG 2020+4017, the flux should lie between these two values.

When modeling the X-ray and gamma-ray spectra from the putative pulsar, we estimate the distance as $1.6 \mathrm{kpc}$, the column density $N_{\mathrm{H}}=1.0 \times 10^{22} \mathrm{~cm}^{-2}$, and $\Gamma_{\mathrm{X}} \sim 1.7$ (Weisskopf et al. 2006). By comparing the estimated spectra with the observed one, we argue that the inclination angle of this putative pulsar lies between $20^{\circ}$ to $30^{\circ}$, here $\alpha=25^{\circ}$. We assume that the characteristic age of the pulsar is similar to that of SNR G78.2+21, 5400 yr (Sturner \& Dermer 1995), which can give us information for constraining the values of period $(P)$ and magnetic field $(B)$. In Fig. 1c, we present the estimated high-energy nonthermal spectrum of the putative pulsar and the observed spectrum of 3EG J2020+4017. The high-energy radiation can be roughly explained when we take the period from $P=0.13$ s to $P=0.19 \mathrm{~s}$, and the corresponding magnetic field changes from $B_{12}=7 \mathrm{G}$ to $B_{12}=10.4 \mathrm{G}$. We also give the estimated spectra when $P=0.16 \mathrm{~s}, B_{12}=8.8 \mathrm{G}$. The gamma-ray beaming solid angle is taken as $\Delta \Omega=0.9$ sr. From Fig. 1c, we can see that a putative pulsar with reasonable spin parameters can explain the observed nonthermal high-energy radiation. However, we cannot precisely estimate the spin parameters of the pulsar because of the uncertainties of both the X-ray spectrum and the inclination angle.

\subsection{The cases of 3EG J0616-3310 and 3EG J1249-8330}

Other than these three promising candidates of Gemingalike gamma-ray pulsars, there are also other candidates that coincident with some unidentified gamma-ray sources, like 3EG J0616-3310 and 3EG J1249-8330 (La Palombara et al. 2006). The gamma-ray fluxes at energy greater than $100 \mathrm{MeV}$ are characterized by power-law spectra with spectral indexes $\Gamma=2.11 \pm 0.24$ for 3 EG J0616-3310 and $\Gamma=2.14 \pm 0.27$ for 3EG J1249-8330, respectively (Hartman et al. 1999). There 
are no radio counterparts that contradict the extragalactic origin. The properties of these two sources show that they could be good candidates for radio-quiet gamma-ray pulsars, so La Palombara et al. (2006) applied a multifrequency method to searching for the counterparts. La Palombara et al. (2006) find that three point sources, XMMU J061807.6-331237, XMMU J061429.8333225 and XMMU J061450.2-331501, are promising candidates for 3EG J0616-3310. But 3EG J1249-8330 has only one possible candidate counterpart, XMMU J124642.5-832212. La Palombara et al. (2006) also estimate the X-ray flux of these sources in the energy range $0.3-10 \mathrm{keV}$ by assuming a powerlaw spectral index $\Gamma_{\mathrm{X}}=1.7$

Here we combine the derived X-ray spectra given by La Palombara et al. (2006), together with the EGRET gamma-ray spectra (Hartman et al. 1999), to model the observed $\mathrm{X}$-ray and gamma-ray spectra of the putative pulsars corresponding to 3EG J0616-3310 and 3EG J1249-8330. Because these two sources are likely to be associated with Gould belt, we assume that the distance of the putative pulsars is $400 \mathrm{pc}$, which is roughly the radius of the Gould belt. We assume the common parameters as $\Delta \Omega=3 \mathrm{sr}, x_{\max }=2.5$, and $\alpha=25^{\circ}$. For XMMU J061807.6-331237, the estimated period $P \sim 0.05-$ $0.07 \mathrm{~s}$, and magnetic field $B_{12} \sim 0.06-0.1 \mathrm{G}$, we present the estimated spectra in Fig. 1d (solid line) when taking $P=0.06 \mathrm{~s}$ and $B_{12}=0.08 \mathrm{G}$, and the characteristic age is $\tau_{\mathrm{c}} \sim 9 \times 10^{6} \mathrm{yr}$. For XMMU J061450.2-331501, the estimated period $P \sim 0.04-$ $0.06 \mathrm{~s}$ and magnetic field $B_{12} \sim 0.05-0.11$ G. In Fig. $1 \mathrm{~d}$, we give the spectrum (dashed line) when taking $P=0.05 \mathrm{~s}$ and $B_{12}=0.08 \mathrm{G}$, and the characteristic age is about $\tau_{\mathrm{c}} \sim$ $6.3 \times 10^{6}$ yr. For XMMU J061429.8-333225, the estimated period $P \sim 0.03-0.05 \mathrm{~s}$, and magnetic field $B_{12} \sim 0.04-0.12 \mathrm{G}$, we present the estimated spectra in Fig. 1d (dotted line) when taking $P=0.04 \mathrm{~s}$ and $B_{12}=0.08 \mathrm{G}$, and the characteristic age is about $\tau_{\mathrm{c}} \sim 4.1 \times 10^{6} \mathrm{yr}$.

Assuming XMMU J124642.5-832212 is the gamma-ray pulsars corresponding to 3EG J1249-8330, the estimated period $P \sim 0.05-0.1 \mathrm{~s}$ and magnetic field $B_{12} \sim 0.08-0.3 \mathrm{G}$. In Fig. 1e, we present the spectra when $P=0.08, B_{12}=0.2$, here we take $\alpha \sim 25^{\circ}$, the gamma-ray beaming solid angle $\Delta \Omega \sim 3 \mathrm{sr}$, and $x_{\max } \sim 2.5$. The characteristic age is $\sim 2.5 \times 10^{6} \mathrm{yr}$. We also present the estimated spectra when $P=0.1 \mathrm{~s}, B_{12}=0.3 \mathrm{G}$, and $P \sim 0.05, B_{12} \sim 0.08$, and the corresponding characteristic ages are $\tau_{\mathrm{c}} \sim 1.8 \times 10^{6} \mathrm{yr}$ and $\sim 6.3 \times 10^{6} \mathrm{yr}$, respectively.

From these calculations, we can see that gamma-ray pulsars with reasonable parameters can explain the observed gamma-ray spectra from 3EG J0616-3310 and 3EG J1249-8330. We argue that the gamma-ray pulsars are mature pulsars with characteristic ages of several million years and with weaker magnetic field compared to young gamma-ray pulsars. Here we can only give a simply estimation about the spin parameters because of insufficient observation of X-ray and gamma-ray emission. More sensitive and precise observations are required for a more reasonable estimation.

\section{Conclusion and discussion}

Based on the revised outer gap model (Zh04), we studied the high-energy, non-thermal radiation from X-ray to $\gamma$ ray bands of five putative Geminga-like gamma-ray pulsars, 3EG J0010+7309, 3EG J1835+5918, 3EG J2020+4017, 3EG J0616-3310, and 3EG J1249-8330. Combining the observed X-ray spectra by Chandra or XMM-Newton and the gamma-ray spectra by EGRET, we estimated the reasonable spin parameters (in Table 1) of the putative pulsars and explain the observed high energy spectra.

For the unidentified EGRET sources, it is important to find counterparts. Pulsars are the only identified Galactic EGRET sources. For the young radio pulsars associated with unidentified sources discovered after the EGRET mission, GLAST will be able to tell us something about the relation of pulsars with these unidentified sources. For other unidentified sources with gamma-ray spectra similar to the gamma-ray pulsars, if the gamma-rays come from Geminga-like gamma-ray pulsars, this process is more difficult because we cannot obtain information from the radio observation. X-ray observations have discovered some radio-quiet compact objects that are spatially coincident with some unidentified gamma-ray sources. It is not clear whether these sources are intrinsically radio weak/quiet or this occurs just because of the beaming effects. In outer gap and polar gap models, the predicted ratio of radio-loud to radio-quiet gamma-ray pulsars is significantly different (Jiang \& Zhang 2006; Harding et al. 2007), and the future observations by GLAST will give more valuable information about the nature of the unidentified gamma-ray sources and improve the models of gamma-ray pulsars.

Acknowledgements. We thank the anonymous referee for her/his very constructive comments. This work is partially supported by a Grant for Distinguished Young Scientists from the NSFC (10425314), Grants from NSFCs (10743001, 10778702), and a Grant from Yunnan Province (2006A0001Q).

\section{References}

Becker, W., Weisskopf, M. C., Arzoumanianet, Z., et al. 2004, ApJ, 615, 897 Brazier, K. T. S., Kanbach, G., Carramiñana, A., et al. 1996, MNRAS, 281, 1033 Brazier, K. T. S., Reimer, O., Kanbach, G., et al. 1998, MNRAS, 295, 819 Cheng, K. S., \& Zhang, L. 1999, ApJ, 515, 337

Cheng, K. S., Ho, C., \& Ruderman, M. A. 1986a, ApJ, 300, 500 (CHR86a) Cheng, K. S., Ho, C., \& Ruderman, M. A. 1986b, ApJ, 300, 522 (CHR86b) Cheng, K. S., Ruderman, M. A., \& Zhang, L. 2000, ApJ, 537, 964 Daugherty, J. K., \& Harding, A. K. 1996, ApJ, 458, 278

Harding, A. K., Grenier, I. A., \& Gonthier, P. L. 2007, Ap\&SS, 309, 221 Harding, A. K., \& Zhang, B. 2001, ApJ, 548, L37

Halpern, J. P., Gotthelf, E. V., Mirabal, N., et al. 2002, ApJ, 573, L41 Halpern, J. P., Gotthelf, E. V., Carmilo, F., et al. 2004, ApJ, 612, 398 Halpern, J. P., Carmilo, F., \& Gotthelf, E. V. 2007, ApJ, 668, 1154 Hartman, R. C., Bertsch, D. L., Bloom, S. D., et al. 1999, ApJS, 123, 79 Hirotani, K. 2007, ApJ, 662, 1173

Hirotani, K., \& Shibata, S. 2001, ApJ, 558, 216

Jiang, Z. J., \& Zhang, L. 2006, ApJ, 643, 1130

Kramer, M., Bell, J. F., Manchester, R. N., et al. 2003, MNRAS, 342, 1299

La Palombara, N., Mignani, R. P., Hatziminaoglou, E., et al. 2006, A\&A, 458, 245

Lamb, R. C., \& Macomb, D. J. 1997, ApJ, 488, 872

Merck, M., Bertsch, D. L., Dingus, B. L., et al. 1996, A\&AS, 120, 465

Mirabal, N., \& Halpern, J. P. 2001, ApJ, 547, L137

Mirabal, N., Halpern, J. P., Eracleous, M., et al. 2000, ApJ, 541, 180

Morrison, R., \& McCammon, D. 1983, ApJ, 270, 119

Muslimov, A. G., \& Harding, A. K. 2004, ApJ, 606, 1143

Pineault, S., Landecker, T. L., Madore, B., et al. 1993, AJ, 105, 1060

Reimer, O., Brazier, K. T. S., Carramiñana, A., et al. 2001, MNRAS, 324, 772

Slane, P. O., Seward, F. D., Bandiera, R., et al. 1997, ApJ, 485, 221

Slane, P., Zimmerman, E. R., Hughes, J. P., et al. 2004, ApJ, 601, 1045

Sturner, S. J., \& Dermer, C. D. 1995, A\&A, 293, L17

Tang, A. P. S., Takata, J., Jia, J. J., \& Cheng, K. S. 2008, ApJ, 676, 562

Takata, J., \& Chang, H. K. 2007, ApJ, 670, 677

Thompson, D. J., Bailes, M., Bertsch, D. L., et al. 1999, ApJ, 516, 297

Weisskopf, M. C., Swartz, D. A., Carraminana, A., et al. 2006, ApJ, 652, 387

Zhang, B., \& Harding, A. K. 2000, ApJ, 532, 1150

Zhang, L., \& Cheng, K. S. 1997, ApJ, 487, 370 (ZC97)

Zhang, L., \& Cheng, K. S. 1998, A\&A, 335, 234

Zhang, L., \& Jiang, Z. J. 2005, ApJ, 632, 523

Zhang, L., \& Jiang, Z. J. 2006, A\&A, 454, 537

Zhang, L., Cheng, K. S., Jiang, Z. J., et al. 2004, ApJ, 335, 234 (Zh04) 